\title{
Pratiques
}

Linguistique, littérature, didactique

161-162 | 2014

Écrire/faire écrire

\section{Séquence didactique axée sur les stratégies et amélioration d'un texte explicatif de comparaison chez des élèves canadiens en milieu minoritaire}

A Strategy-Based Didactic Sequence and Improvement in Linguistic-Minority

Canadian Students' Comparative Expository Texts

\section{Martine Cavanagh}

\section{OpenEdition}

\section{Journals}

Édition électronique

URL : http://journals.openedition.org/pratiques/2187

DOI : $10.4000 /$ pratiques. 2187

ISSN : 2425-2042

Éditeur

Centre de recherche sur les médiations (CREM)

Édition imprimée

Date de publication : 5 juin 2014

Référence électronique

Martine Cavanagh, «Séquence didactique axée sur les stratégies et amélioration d'un texte explicatif de comparaison chez des élèves canadiens en milieu minoritaire », Pratiques [En ligne], 161-162 | 2014, mis en ligne le 05 juin 2014, consulté le 19 avril 2019. URL : http://journals.openedition.org/ pratiques/2187 ; DOl : 10.4000/pratiques.2187

Ce document a été généré automatiquement le 19 avril 2019.

(c) Tous droits réservés 


\title{
Séquence didactique axée sur les stratégies et amélioration d'un texte explicatif de comparaison chez des élèves canadiens en milieu minoritaire
}

\author{
A Strategy-Based Didactic Sequence and Improvement in Linguistic-Minority \\ Canadian Students' Comparative Expository Texts
}

Martine Cavanagh

\section{Introduction}

1 L'écriture est une habileté fort complexe qui s'avère encore plus difficile à maitriser pour les élèves franco-canadiens scolarisés dans les provinces canadiennes hors Québec où le français est la langue de la minorité. En effet, les études indiquent clairement que ces élèves ont tendance à produire des textes « décousus » et à mobiliser des stratégies peu efficaces (Blain, 2001; CMEC, 2004; Cavanagh, 2007a). Ces difficultés résulteraient, en partie du moins, d'une part, d'un enseignement traditionnel mettant davantage l'accent sur le texte en tant que produit fini que sur la démarche intellectuelle qui sous-tend sa rédaction et, d'autre part, d'un manque de ressources pédagogiques et de formation pour apprendre à enseigner de manière explicite les connaissances textuelles et stratégiques s'avérant particulièrement importantes dans les milieux où la langue d'instruction est la langue de la minorité (Cormier, 2005 ; Ball, 2006 ; Cavanagh \& Blain, 2009).

2 Face à ce constat, dans la foulée des travaux effectués par des chercheurs américains (Graham, 2006), nous avons élaboré deux séquences didactiques, l'une afin de soutenir les élèves du primaire dans la production d'un texte d'opinion (Cavanagh, 2005), et l'autre pour les soutenir dans la rédaction d'un récit (Cavanagh, 2007b). Ces séquences, conçues à 
partir d'un ensemble de principes issus de la psychologie cognitive et du socioconstructivisme (Cavanagh, 2010b), placent l'élève au cœur de son apprentissage en l'outillant en stratégies cognitives. Les résultats encourageants obtenus au niveau de la cohérence textuelle (Cavanagh, 2006, 2009), nous ont motivée à concevoir une nouvelle séquence pour permettre aux élèves du secondaire de développer leur capacité à rédiger un texte explicatif.

3 Le texte explicatif a été retenu ici en raison de son importance pour l'apprentissage des matières autres que le français. En effet, l'élève du secondaire est souvent amené à lire et à rédiger des explications pour construire les connaissances conceptuelles et lexicales spécifiques à la matière qu'il étudie, notamment en sciences et en sciences sociales. Ainsi, en raison de sa fonction épistémique, ce type de texte est un outil indispensable au service du développement cognitif de l'élève (Hamman \& Stevens, 2003). Et pourtant, malgré son rôle crucial dans la formation des élèves, les enseignants semblent peu se préoccuper de développer les habiletés nécessaires à sa production (Gagnon \& Ziarko, 2009).

4 Le peu d'attention accordé au texte explicatif se retrouve également au niveau de la recherche en didactique de l'écriture. Du côté des recherches américaines, les métaanalyse de S. Graham (2006) et de S. Graham et D. Perrin (2007) révèlent qu'il existe peu d'études sur le texte explicatif : sur les 39 études répertoriées, 28 d'entre elles portent sur le texte narratif et sur le texte argumentatif. Du côté des recherches en français, les études portant sur le texte explicatif sont encore plus rares. Les travaux d'A. Robert (2001) et de C. Garcia-Debanc (1988) ont mis en évidence les opérations impliquées dans l'écriture d'explications en sciences. À partir des premières propositions de GarciaDebanc, des groupes de recherche ont travaillé sur ce type de texte au primaire et ont fourni aux enseignants des pistes de travail spécifiques surtout du point de vue de la réécriture (Groupe EVA, 1991, 1996; Garcia-Debanc, Planes \& Roger, 2004). L'étude de R. Gagnon et H. Ziarko (2009) a montré les effets positifs d'une séquence didactique visant l'enseignement de la structure du texte et des procédés linguistiques pour linéariser un texte documentaire de comparaison sur les performances d'écriture d'élèves de deuxième année du primaire. À part ces travaux, il n'existe à notre connaissance aucune étude sur ce sujet au niveau du secondaire.

5 Le présent article a pour objectif d'apporter une pièce au corpus d'études sur l'appropriation du texte explicatif en classe en présentant les résultats issus de l'expérimentation d'une séquence didactique visant la production d'un texte explicatif de comparaison auprès d'élèves du secondaire. La variable enseignement a été manipulée de façon à examiner ses effets sur la cohérence des textes produits par les élèves.

\section{Cadre conceptuel}

\section{La définition du texte explicatif}

6 J.-M. Adam (2005), dont la typologie est basée sur les caractéristiques structurales et linguistiques des textes, identifie cinq types de texte (ou séquences prototypiques), à savoir les textes narratif, argumentatif, dialogal, descriptif et explicatif. Les textes de type descriptif et explicatif visent à fournir au lecteur des informations précises, réelles et vérifiables sur un sujet tout en restant neutres et en évitant de donner une opinion personnelle. Pour ce faire, ils contiennent un vocabulaire spécialisé lié spécifiquement au 
sujet traité dans le texte. Mais qu'est-ce qui distingue le texte descriptif du texte explicatif ? Essentiellement, le texte descriptif a pour but de décrire une réalité (un objet, un être, un évènement, etc.) en nommant ses propriétés, ses qualités et ses parties (il répond aux questions quoi? et comment?) tandis que le texte explicatif vise à faire comprendre une affirmation, un fait ou un phénomène à quelqu'un (il répond à la question pourquoi ?). Au plan cognitif, le texte explicatif est plus contraignant que le texte descriptif, car il exige que les élèves explicitent des liens explicatifs plutôt que de simplement juxtaposer des informations (Garcia-Debanc, 1988).

\section{Le concept de cohérence textuelle en lien avec le texte explicatif de comparaison}

7 Les travaux menés depuis les années 70 en grammaire du texte ont mis en lumière le fait que la qualité d'un texte dépend en grande partie de sa cohérence. Toutefois, la notion de cohérence est difficile à cerner. Il en existe plusieurs définitions pouvant se regrouper dans deux catégories, à savoir la cohérence "dérivée » et la cohérence "explicite " (Carter-Thomas, 2000 : 33). La cohérence dérivée met l'accent sur le lecteur. Elle résulte de l'interaction entre le texte et le lecteur qui met tout en œuvre pour établir des liens qui ne figurent pas forcément dans le texte. Dans ce cas, la cohérence est surtout une question de réception textuelle (Adam, 2008). À l'inverse, la cohérence explicite souligne l'importance du travail du scripteur qui s'efforce de faciliter la tâche interprétative du lecteur en le guidant grâce à des marques d'organisation du texte et à des éléments de textualisation explicite. Puisque notre étude se situe dans un contexte scolaire où il s'agit d'amener les élèves à rédiger des textes explicitement cohérents, nous choisissons de nous appuyer sur la seconde définition de la cohérence. La séquence expérimentée ici vise donc la production par les élèves d'un texte explicatif comportant des évocations explicites de sa cohérence à la fois macrostructurelle, microstructurelle et situationnelle.

\section{La cohérence macrostructurelle}

8 Liée à la notion de types de textes (Adam, 2005), la cohérence macrostructurelle concerne l'organisation du texte dans sa globalité. Elle résulte de la capacité du scripteur à découper son texte en paragraphes selon la structure du type de texte visé tout en signalant le passage d'un paragraphe à l'autre à l'aide d'organisateurs textuels. La macrostructure du texte explicatif comporte généralement une phase introductive de questionnement qui présente le phénomène à expliquer, une phase explicative qui expose les éléments de l'explication et une phase conclusive qui résume l'explication (Grenier, 2008). Dans la phase explicative, l'organisation des idées varie selon la nature de la relation mise en évidence (Giasson, 2007). Ainsi, l'explication peut être présentée selon une structure cause-conséquence, problème-solution et comparaison. Pour la présente étude, nous avons retenu la structure de comparaison, car elle s'avère plus contraignante que les deux autres en raison de la complexité et de la simultanéité des processus qui entrent en jeu dans le traitement en alternance de deux réalités (Meyer, 1985 ; McArthur \& Philippakos, 2010). Selon N.-N. Spivey (1991), il existe trois façons de structurer une comparaison : 1) par sujets (par exemple, tout sur l'ours brun et tout sur les ours noirs); 2) par aspects (par exemple, la nourriture, l'habitat); et 3) selon les ressemblances et des différences. Les élèves de notre étude étant peu familiers avec la structure de comparaison, nous avons privilégié le troisième type d'organisation considéré comme 
étant plus facile que les deux premiers, car il implique un nombre plus restreint d'éléments à mettre en parallèle (Richgels, McGee, Lomax \& Sheard, 1987).

\section{La cohérence microstructurelle}

La cohérence microstructurelle se joue au niveau de la phrase. Elle découle de la capacité du scripteur à faire progresser son texte grâce à l'apport constant d'informations nouvelles (Charolles, 1978, 2002, 2006), mais tout en assurant la continuité des idées d'une phrase à l'autre (De Weck, 1991; Charolles, 2002, 2006) et en explicitant le rapport logique entre les idées (Lundquist, 1980). Chacune de ces trois opérations fait appel à des procédés linguistiques spécifiques. Dans le contexte de la séquence expérimentée ici, nous avons regroupé les procédés de progression dans deux catégories : ceux qui servent à donner un ton objectif au texte explicatif (par exemple, les définitions, les données chiffrées), et ceux qui rendent le texte moins « sec » en le personnalisant (par exemple, la projection et la supposition). Les procédés de continuité, quant à eux, comprennent les pronoms, les substituts nominaux dont les synonymes et la répétition d'un même terme. Bien utilisés, ils jouent un rôle important dans le texte de comparaison, car ils permettent au lecteur de repérer sans ambiguïté à quel élément comparé le scripteur réfère. Enfin, l'explicitation des rapports logiques entre les idées passe par l'utilisation de connecteurs tels que donc et ainsi pour mettre en relief le raisonnement qui sous-tend l'explication ou par contre et tandis que pour signaler une relation d'opposition tout en introduisant le second terme de la comparaison. Ces deux types de connecteurs ont fait l'objet d'un enseignement dans le cadre de la séquence sur le texte explicatif.

\section{La cohérence situationnelle}

10 Une dernière dimension de la cohérence qu'il importe de prendre en considération concerne le rapport qui existe entre le texte et la situation d'écriture qui l'a généré. En effet, le scripteur qui rédige un texte explicatif doit tenir compte de l'intention d'écriture qu'il poursuit et des connaissances lexicales et conceptuelles du destinataire. Dans cette optique, il lui faut évaluer le besoin de définir certains termes plus spécialisés et adapter le vocabulaire et les tournures syntaxiques pour qu'elles soient à la portée du lecteur. Sur le plan des connaissances conceptuelles, il doit s'assurer de fournir des informations nouvelles et intéressantes pour le destinataire et éviter de donner des détails qui sont en contradiction avec la réalité évoquée (Charolles, 1978). Cette cohérence dite situationnelle (Apotheloz \& Mieville, 1989), qui touche à la pertinence du contenu en fonction des paramètres de la situation d'écriture et à celle des ressources linguistiques mobilisées pour en rendre compte, a également fait partie des apprentissages visés par la séquence expérimentée dans le cadre de la présente recherche.

\section{Les stratégies cognitives en lien avec le texte explicatif de comparaison}

11 Les travaux en psychologie cognitive portant sur le fonctionnement du scripteur novice et du scripteur expert ont montré que, chez le scripteur habile, la production d'un texte cohérent dépend, en grande partie, de processus cognitifs de haut niveau dont l'activation passe par l'emploi de stratégies efficaces. Par stratégie, on entend «l'ensemble des activités cognitives et comportementales potentiellement conscientes 
choisies ou construites par l'apprenant dans le but de contrôler, soit directement, soit indirectement, les processus cognitifs qui interviennent dans la réalisation d'une tâche d'apprentissage » (Hensler, 1992: 41). Les stratégies d'écriture sont généralement spécifiques au type de texte visé et elles sont réparties en fonction des trois processus cognitifs identifiés par J. Hayes $(1995,1998,2004)$, soit la planification, la mise en texte et la révision. Ces travaux, ainsi que ceux qui rendent comptent des difficultés des élèves en matière d'écriture de textes explicatifs (Laparra, 1986) nous ont inspirée dans la conception de dix stratégies pour soutenir les élèves dans la production d'un texte explicatif de comparaison. Pour concrétiser ces stratégies pour les élèves, en plus de les représenter visuellement sur des feuilles aide-mémoire, nous les avons associées à un pictogramme et les avons nommées de manière à suggérer, par le biais d'une analogie, leur fonction spécifique à l'intérieur du processus d'écriture dans lequel elles s'insèrent (tableau 1).

Tableau 1. Les dix stratégies pour apprendre à écrire un texte explicatif de comparaison (Cavanagh, 2010a).

\begin{tabular}{|c|c|c|c|c|c|c|c|}
\hline \multirow[b]{2}{*}{ Stratégies } & \multirow[b]{2}{*}{ Pictogrammes } & \multicolumn{2}{|c|}{ Projet 1} & \multicolumn{2}{|c|}{ Projet 2} & \multicolumn{2}{|c|}{ Projet 3} \\
\hline & & $\begin{array}{c}\text { Je coche } \\
\text { ici }\end{array}$ & $\begin{array}{c}\text { Initiales } \\
\text { de mon } \\
\text { enseignant }\end{array}$ & $\begin{array}{c}\text { Je coche } \\
\text { ici }\end{array}$ & $\begin{array}{c}\text { Initiales } \\
\text { de mon } \\
\text { enseignant }\end{array}$ & $\begin{array}{c}\text { le coche } \\
\text { ici }\end{array}$ & $\begin{array}{l}\text { Initiales } \\
\text { de mon } \\
\text { enseignant }\end{array}$ \\
\hline $\begin{array}{l}\text { 1. Faire un choix } \\
\text { éclairé }\end{array}$ & & & & & & & \\
\hline $\begin{array}{l}\text { 2. Examiner } \\
\text { à la loupe }\end{array}$ & & & & & & & \\
\hline $\begin{array}{l}\text { 3. Cibler les } \\
\text { informations }\end{array}$ & & & & & & & \\
\hline $\begin{array}{l}\text { 4. Survoler } \\
\text { I'information }\end{array}$ & (2) & & & & & & \\
\hline $\begin{array}{l}\text { 5. Noter } \\
\text { I'essentiel }\end{array}$ & & & & & & & \\
\hline $\begin{array}{l}\text { 6. Jeter un } \\
\text { coup d'oeil }\end{array}$ & & & & & & & \\
\hline $\begin{array}{l}\text { 7. Développer une } \\
\text { idée principale } \\
\text { (DIP) }\end{array}$ & & & & & & & \\
\hline $\begin{array}{l}\text { 8. Apporter } \\
\text { avec moi }\end{array}$ & & & & & & & \\
\hline $\begin{array}{l}\text { 9. Mieux voir } \\
\text { mes idées }\end{array}$ & 8 & & & & & & \\
\hline $\begin{array}{l}\text { 10. Vérifier } \\
\text { mon texte }\end{array}$ & & & & & & & \\
\hline
\end{tabular}

Parmi les dix stratégies enseignées, six d'entre elles guident l'élève dans la planification, deux stratégies l'aident au moment de la mise en texte et les deux autres le soutiennent dans la révision. Chaque stratégie agit sur une ou plusieurs dimensions de la cohérence textuelle.

\section{Les stratégies de planification}

13 À l'étape de la planification, les deux premières stratégies sont liées au traitement de la situation d'écriture. Ainsi, la stratégie "Faire un choix éclairé ", incite l'élève à se questionner par rapport aux diverses situations d'écriture qui lui sont proposées afin qu'il parvienne à faire un choix judicieux basé sur ses intérêts et ses connaissances. Avec la stratégie "Examiner à la loupe ", il apprend à analyser la situation choisie en s'interrogeant sur les connaissances du destinataire, l'intention d'écriture et le type de structure à adopter. Elle amène l'élève à préciser les exigences de la tâche et le conduit à 
se fixer des buts qui orienteront sa recherche d'information. Il en résultera une meilleure cohérence situationnelle au niveau du texte produit.

Une fois la tâche clarifiée, comme le texte explicatif repose sur des connaissances spécialisées, l'élève doit passer à l'étape de la recherche d'informations dans plusieurs sources documentaires, ce qui l'oblige à mobiliser des stratégies de lecture (Cartier, 2007). La séquence didactique prévoit donc l'enseignement de trois stratégies de lecture. La première, intitulée "Cibler les informations", est une stratégie de pré-lecture qui s'inspire de la technique de lecture réflexive développée par D. Ogle (1986) et connue en anglais sous l'acronyme KWL (Know: ce que je sais déjà ; Want to know : ce que je veux savoir et Learned: ce que j'ai appris). Cette stratégie amène l'élève à planifier sa lecture d'un texte documentaire en activant ses connaissances sur les éléments à comparer (qu'est-ce que je sais?) et en se fixant une intention de lecture (qu'est-ce que je veux savoir?). Après la lecture, elle le pousse à évaluer les connaissances qu'il a acquises (qu'est-ce que j'ai appris ? ). Toutefois, la réponse à cette dernière question exige que le scripteur choisisse des documents contenant des informations sur les objets à comparer. Or, ce travail de sélection de documents pertinents n'est pas évident pour le scripteur novice qui, au lieu de recourir à une stratégie d'écrémage (Pigallet, 1996) pour évaluer rapidement l'utilité d'un document, a tendance à se lancer d'emblée dans une lecture en profondeur des documents jugés en apparence utiles en perdant de vue son objectif final. La stratégie " Survoler l'information " permet d'éviter cet écueil en offrant aux élèves une méthode de sélection de documents basée sur l'utilisation des titres et des sous-titres et sur l'identification des mots clés.

Suit la lecture approfondie de chaque document retenu et la sélection d'informations importantes grâce à la stratégie " Noter l'essentiel ». Cette stratégie de prise de notes incite le lecteur à mobiliser trois opérations : 1) repérer les aspects de la comparaison auxquels il avait déjà pensé lors de son emploi de la stratégie «Cibler les informations » et noter les nouveaux détails qui leur sont associés ; 2) noter les aspects nouveaux qu'il a découverts ainsi que les détails qui les accompagnent; et 3) parmi les aspects répertoriés, retenir seulement ceux qui lui seront utiles pour établir la comparaison. Cette stratégie, si importante pour la réussite scolaire des élèves (Falardeau \& Loranger, 1993), permet d'éviter certains pièges dans lesquels tombent souvent les scripteurs novices, à savoir le manque de développement des aspects qui réduit le texte comparatif à une courte liste ennuyeuse, la comparaison de deux aspects de nature différente et l'oubli d'un des deux termes de la comparaison laissant le lecteur dans la confusion. À notre connaissance, ces trois stratégies de lecture n'ont jamais fait partie d'une intervention sur l'écriture. Bien que certains chercheurs (Dickson, 1999; Wong, 2000; Hamman \& Stevens, 2003) aient inclus une stratégie de résumé au sein de leur intervention, celle-ci était plutôt générale et ne soutenait pas les opérations spécifiques à la base de la lecture d'un texte de comparaison.

Une fois le contenu sélectionné, le scripteur passe à son organisation avec l'aide de la stratégie "Jeter un coup d'œil». Cette stratégie, qui prend la forme d'un schéma représentant visuellement la macrostructure du texte explicatif de comparaison (annexe 2), offre au scripteur un cadre pour organiser ses notes (Giasson, 2007). En alliant des informations numériques et des informations analogiques, ce schéma mobilise chez le scripteur deux systèmes de codage, ce qui augmente sa capacité à mémoriser toutes les parties du texte de comparaison et leur agencement dans un tout cohérent (Adam, 1999). Ainsi, par son pouvoir structurant et mnésique, le schéma constitue la stratégie centrale 
et la base sur laquelle se construisent les connaissances sur la cohérence macrostructurelle (Karmiloff-Smith, 1992 ; Brassart, 1998 ; Cavanagh, 2006).

\section{Les stratégies de mise en texte}

17 Le scripteur a maintenant atteint l'étape de la mise en texte proprement dite. À cette étape, le défi pour lui consiste à développer les bribes d'idées répertoriées dans son schéma et à les transformer en phrases complètes qui s'enchainent. Les deux stratégies qui sont mises à sa disposition pour lui permettre de relever ce défi prennent la forme de procédés linguistiques utiles pour réaliser l'une ou l'autre de ces opérations de linéarisation. Ainsi, la stratégie « DIP », acronyme pour donner des détails (D), illustrer (I) et personnaliser $(\mathrm{P})$ consiste en un tableau à trois volets qui rappelle à l'élève qu'il peut développer ses idées de trois manières : 1 ) en donnant des détails sous forme de données chiffrées, de définitions ou de descriptions ; 2) en les illustrant à l'aide d'exemples ou de citations; et 3) en personnalisant par l'ajout de projections et de suppositions. La stratégie "Apporter avec moi » lui fournit des procédés de reprise d'information et lui rappelle qu'à chaque fois qu'il ajoute une nouvelle phrase, il doit apporter avec lui une partie de la phrase précédente afin que ses idées s'enchainent.

Utilisés conjointement avec des connecteurs servant à introduire le second terme de la comparaison, ces procédés permettent au lecteur de savoir en tout temps à quel référent le scripteur fait allusion et sur quel aspect porte la comparaison.

\section{Les stratégies de révision}

19 À la suite de l'étape rédactionnelle survient le processus de la révision qui mobilise l'opération difficile de la lecture critique et nécessite le recours à divers signes (encoches, flèches, etc.) afin de modifier le texte sans avoir à le recopier dans son entier. Ce processus est soutenu par l'emploi de deux stratégies. La première intitulée «Mieux voir mes idées" est symbolisée par des lunettes contenant des questions telles que $\mathrm{Ma}$ comparaison est-elle bien structurée? ou Est-ce que je compare bien le même aspect? Comme dans d'autres recherches (Blain \& Painchaud, 1999; Blain, 2001; Blain \& Lafontaine, 2010), cette stratégie est utilisée dans le cadre d'un travail en dyades où les élèves échangent leur texte et se posent mutuellement des questions afin d'amener leur partenaire à clarifier ses idées. La seconde stratégie prend la forme d'une grille de vérification qui encourage le scripteur à relire son texte et à l'améliorer à la lumière des critères de qualité d'un texte de comparaison qu'il aura construits au fil de sa participation aux diverses tâches prévues dans la séquence.

Pour faciliter l'appropriation des stratégies par les élèves et l'atteinte de leur autonomie cognitive, la séquence comporte différentes formes d'échafaudage (Vygotsky, 1978) ou de soutien progressivement retiré selon les besoins particuliers des élèves. Parmi elles, comme dans les nombreuses études rapportées par S. Graham (2006) et S. Graham et K.R. Harris (2003), le modelage, c'est-à-dire la verbalisation par l'enseignante de ses processus de pensée tandis qu'elle emploie les stratégies devant les élèves lors de l'écriture de son propre texte, occupe une place importante dans le dispositif didactique.

21 Notre étude avait pour objectif d'examiner les effets d'une séquence didactique visant à outiller les élèves en stratégies sur la capacité d'élèves francophones du secondaire à rédiger des textes explicatifs de comparaison cohérents. En lien avec cet objectif général, nous avons formulé l'hypothèse prédisant qu'une intervention axée sur l'enseignement 
de plusieurs stratégies spécifiques à l'écriture d'un texte explicatif de comparaison permettrait aux élèves d'améliorer leur texte au niveau des trois dimensions de la cohérence textuelle.

\section{Méthodologie}

\section{Plan de recherche et échantillon}

Nous avons évalué les effets de la séquence selon un plan de recherche prétest/ traitement/post-test soumis à un groupe expérimental et à un groupe témoin. Le prétestet le post-test ont mesuré la qualité du texte des élèves du point de vue des trois dimensions de la cohérence textuelle. De plus, pour mieux interpréter les résultats quantitatifs, nous avons mené des entrevues auprès de l'enseignante qui a expérimenté la séquence dans sa classe.

Étant donné que les participants n'ont pas été choisis au hasard (Boudreault, 2000), cette recherche est qualifiée de quasi expérimentale. L'échantillon comprenait 81 élèves âgés de 12 ans et répartis dans quatre classes situées dans une école francophone du Conseil scolaire Centre-nord à Edmonton en Alberta. Les participants de deux classes (soit 40 sujets) ont formé le groupe expérimental, tandis que les sujets des deux autres classes ont constitué le groupe témoin (soit 41 sujets). Chaque groupe, qui avait un enseignant différent, comprenait la même proportion garçons/filles (22 filles et 18 garçons pour le groupe expérimental et 21 filles et 20 garçons pour le groupe témoin). De plus, selon les résultats obtenus en français écrit, tel qu'indiqué sur le bulletin scolaire de l'année scolaire précédente, les deux classes contenaient une proportion quasi équivalente d'élèves forts, moyens et faibles.

\section{La séquence didactique}

Concernant le traitement, les élèves du groupe témoin ont bénéficié de l'enseignement habituel de leur enseignante qui a consisté à lire des textes explicatifs pour en dégager les composantes et à en rédiger trois, mais sans recevoir d'enseignement sur les stratégies sous-tendant leur production. Pour les élèves du groupe expérimental, le traitement a pris la forme d'une séquence axée sur l'enseignement explicite des stratégies décrites plus haut. Cette séquence était composée de 15 leçons (Cavanagh, 2010a), d'une durée approximative de 50 minutes chacune, et réparties dans les trois phases de l'enseignement stratégique (Tardif, 1992, 1999), soit les phases de préparation, de réalisation et d'intégration des apprentissages (tableau 2).

Tableau 2. Synthèse de la séquence didactique sur le texte explicatif de comparaison.

\begin{tabular}{|c|c|c|}
\hline Phases & Étapes & Leçons \\
\hline $\begin{array}{l}\text { Préparation des } \\
\text { apprentissages }\end{array}$ & $\begin{array}{lr}\text { Motivation } & \text { et } \\
\text { activation } & \text { des } \\
\text { connaissances } & \end{array}$ & $\begin{array}{l}\text { 1. Bilan des acquis et identification d'un } \\
\text { projet d'écriture }\end{array}$ \\
\hline
\end{tabular}




\begin{tabular}{|c|c|c|}
\hline & $\begin{array}{llr}\text { Construction } & & \text { de } \\
\text { connaissances } & \text { sur } & \text { le } \\
\text { produit fini } & & \end{array}$ & $\begin{array}{l}\text { 2. Analyse d'un texte bien construit } \\
\text { 3. Analyse d'un texte mal construit }\end{array}$ \\
\hline $\begin{array}{l}\text { Réalisation } \\
\text { apprentissages } \\
\text { «Encodage» }\end{array}$ & $\begin{array}{l}\text { Construction de } \\
\text { connaissances sur la } \\
\text { démarche } \\
\text { rédactionnelle } \\
\text { Modelage et pratique } \\
\text { guidée } \\
1^{\text {er }} \text { projet d'écriture }\end{array}$ & $\begin{array}{l}4 \text { à } 12 . \text { Modelage des } 10 \text { stratégies suivi } \\
\text { d'une pratique guidée } \\
\text { (l'élève dispose d'une pochette } \\
\text { informative). }\end{array}$ \\
\hline $\begin{array}{l}\text { Intégration des } \\
\text { apprentissages } \\
\text { "Recontextualisation et } \\
\text { décontextualisation " }\end{array}$ & $\begin{array}{l}\text { Pratique autonome } \\
2^{\mathrm{e}} \text { projet d'écriture }\end{array}$ & $\begin{array}{l}13 \text { à } 15 . \text { Planification, rédaction et } \\
\text { révision d'un } 2^{\mathrm{e}} \text { texte à l'aide des } \\
\text { stratégies apprises au cours de la phase } \\
\text { de réalisation des apprentissages } \\
\text { (L'élève ne dispose pas d'une pochette } \\
\text { informative. Il doit chercher lui-même } \\
\text { les sources d'information). }\end{array}$ \\
\hline
\end{tabular}

Durant la phase de préparation des apprentissages (leçon 1), les élèves ont rédigé individuellement un premier texte de comparaison (pré-test) qui a fait ensuite l'objet d'une confrontation en petits groupes dans le but de faire ressortir leurs connaissances antérieures sur les caractéristiques de ce type de texte.

La phase de réalisation des apprentissages (leçons 2 à 12) a été divisée en deux étapes. Durant la première étape (leçons 2 et 3), les élèves ont réalisé plusieurs activités de manipulation telles que l'analyse de textes bien et mal construits à l'aide du schéma représenté à l'annexe 2. Lors de la deuxième étape (leçons 4 à 12), à partir d'une situation d'écriture, l'enseignante a planifié, rédigé et révisé un texte devant les élèves en modelant les stratégies prévues dans la séquence. Le modelage ${ }^{1}$ de chaque stratégie a été suivi d'une pratique guidée durant laquelle les élèves ont, à partir de leur propre situation d'écriture, bâti progressivement leur texte tout en recevant de la rétroaction sur leur emploi des stratégies.

En principe, durant la phase d'intégration des apprentissages (leçons 13 à 15), les élèves auraient dû avoir l'occasion de réinvestir les stratégies apprises en rédigeant un deuxième texte dans le contexte d'une discipline autre que le français. Toutefois, en raison des contraintes de temps, cela n'a pas été possible.

\section{Formation de l'enseignante}

La formation offerte à l'enseignante avant et pendant l'intervention a été inspirée du modèle conçu par P. Beard El-Dinary et T.Schuder (1993). Avant l'intervention, l'enseignante a participé à une journée de formation avec la chercheure afin de se familiariser avec les principes de base et la structure de la séquence didactique sur le texte explicatif de comparaison. Pendant l'intervention, elle a participé à quatre rencontres d'une demi-journée avec la chercheure. Le but de ces rencontres était d'échanger avec la chercheure sur son expérience d'enseignement et de recevoir une 
formation plus pointue sur la façon de mener certaines activités, notamment en ce qui concerne le modelage des stratégies.

\section{Instrument de cueillette de données et déroulement}

Deux instruments de cueillette de données ont été utilisés : les productions écrites des élèves et les entretiens avec l'enseignante. Les productions écrites ont été rédigées lors du pré-test et du post-test. Le pré-test a servi à établir le niveau de base des élèves et le posttest, administré six semaines plus tard, juste après la mise en œuvre de la séquence didactique, a servi à mesurer les progrès réalisés en cohérence textuelle. Lors de ces deux tests, les élèves du groupe témoin et du groupe expérimental ont eu le temps nécessaire pour écrire un texte explicatif de comparaison sur l'une ou l'autre, au choix, de deux situations d'écriture qui leur ont été présentées. Nous avons cherché à réduire le coût cognitif provenant de la complexité de la tâche en proposant aux élèves d'écrire à partir de faits marquants ou provenant de leur environnement familier. Ainsi, lors du pré-test, la première situation d'écriture demandait à l'élève de comparer le football américain et le soccer et, la seconde, l'invitait à comparer les jeux olympiques de Montréal en 1976 à ceux de Vancouver en 2010. Lors du post-test, les élèves devaient comparer soit la province de l'Alberta à celle de Québec, soit la ville de Vancouver en ColombieBritannique à celle d'Edmonton en Alberta. Pour s'assurer que les sujets étaient à la fois appropriés aux intérêts des élèves et d'un niveau de difficulté équivalents, les mesures suivantes ont été prises. D'abord, nous avons consulté les enseignants pour vérifier la pertinence des sujets. Ensuite, nous avons soutenu les élèves dans la collecte d'informations en leur fournissant une pochette informative contenant une variété de documents (articles, tableaux, cartes postales, guides touristiques, forums de discussion, etc.) sur les sujets exposés dans les situations d'écriture. Ainsi, tous les élèves ont rédigé leur texte à partir des mêmes sources d'information.

Durant l'intervention, nous avons mené quatre entretiens avec l'enseignante afin d'obtenir sa rétroaction par rapport à son expérience d'enseignement. En particulier, nous avons noté les problèmes qu'elle a rencontrés lors de l'animation des activités proposées dans la séquence et la façon dont les élèves ont réagi à ces activités (difficultés rencontrées, niveau de motivation, etc.) Les données qualitatives provenant de ces entretiens ont fait l'objet de rapports écrits et ont servi à apporter un éclairage sur certaines données quantitatives.

\section{Méthode d'analyse des données}

31 L'analyse du contenu des textes a été réalisée à l'aide d'une grille de notation qui a mesuré les trois dimensions de la cohérence sur une échelle allant de 0 à 5 pour chacun des aspects considérés. Pour la dimension macrostructurelle, les aspects suivants ont été pris en compte : 1) la présence d'une introduction comprenant un sujet amené, un sujet posé et un sujet divisé ; 2) la présence d'un paragraphe traitant des différences ; 3) la présence d'un paragraphe traitant des ressemblances; 4) la présence d'une conclusion comprenant un résumé et une ouverture et 5) la présence d'organisateurs textuels pour marquer la transition entre le paragraphe des ressemblances et celui des différences.

Les aspects liés à la cohérence microstructurelle concernaient : 1) la progression des idées dans le paragraphe des ressemblances et dans celui des différences par l'ajout 
d'informations nouvelles, mais sans se répéter ni digresser ; 2) la continuité des idées par l'emploi de procédés de reprise de l'information et 3) l'expression des rapports logiques entre les idées grâce à l'utilisation de connecteurs soutenant spécifiquement l'organisation des idées selon une comparaison.

Quant à la cohérence situationnelle, elle a été évaluée en examinant la capacité de l'élève à faire trois choses: 1) respecter les contraintes imposées par la situation d'écriture; 2) développer ses idées en incorporant des informations intéressantes et objectives et en recourant aux procédés linguistiques suggérés par la stratégie «DIP » et 3) utiliser un vocabulaire précis, voire même spécialisé, qui confère au texte un ton sérieux. Pour assurer l'objectivité du codage à l'aide de ces grilles, nous avons eu recours à la méthode inter-juges. Deux juges qui ne connaissaient ni les participants, ni les objectifs de l'étude ont évalué les productions des élèves. Selon la formule de Spearman, le coefficient d'accord dans les codifications faites par ces juges était de 0.95 pour la macrostructure, 0.80 pour la microstructure et 0.85 pour la dimension situationnelle.

Les différences dans le score global (la somme de toutes les variables mesurées) entre le pré-testet le post-test, pour les deux groupes, ont été analysées à l'aide du test $t$ de Student pour groupes indépendants, les données rencontrant les critères de distribution normale. Par contre, pour analyser les différences obtenues dans chacune des souscatégories, puisque les données étaient discrètes, nous avons utilisé le test non paramétrique de Mann-Whitney (test $\mathrm{U}$ ). Le seuil de signification retenu était $\mathrm{p}<0.05$.

Les données qualitatives recueillies à partir des entretiens, n'ont pas été analysées à partir d'une méthode d'analyse qualitative spécifique. Tel que mentionné plus haut, ces données ont plutôt été utilisées dans le cadre de la discussion des résultats quantitatifs afin de faciliter la compréhension de ceux-ci et d'alimenter la réflexion quant aux hypothèses explicatives et aux pistes à suivre pour favoriser à l'avenir une mise en œuvre plus efficace de la séquence didactique, en particulier pour les élèves plus faibles.

\section{Résultats}

La présentation des résultats porte d'abord sur le score global obtenu par les élèves en cohérence textuelle. Par la suite, les scores globaux et les scores partiels pour chacune des sous-composantes sont examinés séparément.

\section{Résultat global en cohérence textuelle}

37 Le tableau 3 présente la différence moyenne globale et l'écart-type pour les scores obtenus au pré-test et au post-test par les deux groupes expérimental et témoin, ainsi que le résultat du test $t$ sur les différences moyennes entre ces groupes.

Tableau 3. Différences moyennes globales entre le pré-test et le post-test et résultat du test t entre les groupes expérimental et témoin.

\begin{tabular}{|l|l|l|l|l|}
\hline EN & $\begin{array}{l}\text { Groupe expérimental } \\
(\mathrm{n}=40)\end{array}$ & $\begin{array}{l}\text { Groupe témoin } \\
(\mathrm{n}=41)\end{array}$ & Test-t & Prob. \\
\hline Moyenne & 19,65 & $-14,89$ & ${ }_{14,34}$ & $0,000^{* * * 2}$ \\
\hline
\end{tabular}




\begin{tabular}{|c|c|c|}
\hline Écart-type & 12,65 & 9,41 \\
\hline
\end{tabular}

Ainsi, pour l'ensemble des variables mesurées, la différence moyenne entre le pré-test et le post-test est de 19,65 pour le groupe expérimental (écart-type : 12,65) et de -14,89 (écart-type : 9,41) pour le groupe témoin. Cette différence négative pour le groupe témoin montre que les élèves appartenant à ce groupe ont moins bien réussi au post-test qu'au prétest. Le test $t$ sur les différences moyennes entre le groupe expérimental et le groupe contrôle permet de montrer que la différence entre les deux groupes est statistiquement significative $(\mathrm{p}<0.001)$. On peut donc conclure que, de façon générale, pour ce qui est du résultat global, les élèves du groupe expérimental, qui ont bénéficié de l'intervention, ont davantage progressé que les élèves du groupe contrôle. Toutefois, pour déterminer la part de chaque dimension de la cohérence textuelle dans ce résultat global, il nous faut examiner séparément les résultats obtenus pour chacune des dimensions et leurs composantes.

\section{Résultats obtenus au niveau de la cohérence textuelle}

Le tableau 4 présente les différences moyennes obtenues entre le pré-test et le post-test pour les deux groupes expérimental et contrôle dans chacun des aspects de la cohérence textuelle soit la « cohérence macrostructurelle », la « cohérence microstructurelle » et la " cohérence situationnelle ", ainsi que le niveau de signification des différences entre ces groupes, tel que déterminé par le test $\mathrm{U}$ de Mann-Whitney.

Tableau 4. Différences moyennes (écarts-types) entre les résultats au pré-test et au post-test obtenus par les deux groupes en cohérence textuelle, et degrés de signification (p).

\begin{tabular}{|c|c|c|c|}
\hline $\begin{array}{l}\text { Dimensions de la cohérence } \\
\text { textuelle }\end{array}$ & $\begin{array}{l}\text { Groupe } \\
\text { expérimental } \quad(n= \\
40)\end{array}$ & $\begin{array}{l}\text { Groupe } \\
\text { témoin } \quad(n= \\
41)\end{array}$ & $\begin{array}{l}\text { Valeur de p (U de } \\
\text { Mann-Whitney) }\end{array}$ \\
\hline $\begin{array}{l}\text { Cohérence } \\
\text { macrostructurelle } \\
\text { Note globale } \\
\text { Introduction } \\
\text { Développement (différences) } \\
\text { Développement } \\
\text { (ressemblances) } \\
\text { Conclusion } \\
\text { Transitions entre les } \\
\text { paragraphes }\end{array}$ & $\begin{array}{l}10,90(5,81) \\
1,75(1,35) \\
1,25(2,26) \\
3,53(1,40) \\
1,98(1,00) \\
2,40(1,96)\end{array}$ & $\begin{array}{l}-0,10(5,63) \\
-0,54(1,63) \\
-0,51(2,26) \\
0,54(1,76) \\
0,39(1,51) \\
0,00(1,50)\end{array}$ & $\begin{array}{l}0,0000^{* * * 3} \\
0,0005^{* * *} \\
0,0007^{* * *} \\
0,0000^{* * *} \\
0,0000^{* * *} \\
0,0000^{* * *}\end{array}$ \\
\hline $\begin{array}{l}\text { Cohérence } \\
\text { microstructurelle } \\
\text { Note globale } \\
\text { Progression } \\
\text { Continuité } \\
\text { Relation }\end{array}$ & $\begin{array}{l}4,00(3,57) \\
1,50(1,48) \\
1,60(1,32) \\
0,90(1,45)\end{array}$ & $\begin{array}{l}0,49(2,79) \\
0,00(1,31) \\
0,49(1,11) \\
0,00(1,24)\end{array}$ & $\begin{array}{l}0,0000^{* * *} \\
0,0000^{* * *} \\
0,0009^{* * *} \\
0,0129^{* * *}\end{array}$ \\
\hline
\end{tabular}




\begin{tabular}{|l|l|l|l|}
\hline $\begin{array}{l}\text { Cohérence situationnelle } \\
\text { Note globale }\end{array}$ & & \\
Respect des contraintes (détails & $4,75(4,24)$ & $-0,41(4,55)$ & $0,0000^{* * *}$ \\
Développement & $1,90(1,13)$ & $0,07(1,29)$ & $0,0000^{* * *}$ \\
intéressants) & $0,98(1,19)$ & $-0,17(1,20)$ & $0,0000^{* * *}$ \\
Développement (informations & $0,95(1,22)$ & $-0,07(1,29)$ & $0,0004^{* * *}$ \\
objectives) & $0,93(1,23)$ & $-0,24(1,16)$ & $0,0000^{* * *}$ \\
Vocabulaire et ton & & & \\
\hline
\end{tabular}

La lecture de ce tableau montre que si l'on compare les différences entre le pré-test et le post-test pour les deux groupes par rapport à chaque dimension de la cohérence textuelle, toutes les dimensions enregistrent une différence statistiquement significative en faveur du groupe expérimental $(\mathrm{p}<0.001)$. L'hypothèse selon laquelle le programme produirait des changements par rapport à chaque dimension de la cohérence textuelle est donc confirmée.

De façon à pouvoir caractériser précisément les procédés explicatifs utilisés par le groupe expérimental et par le groupe témoin, puisque le texte explicatif consiste essentiellement à faire comprendre un phénomène en présentant des faits vérifiables et objectifs par le biais de procédés pour développer une explication, nous complétons l'analyse statistique liée à la dimension situationnelle et plus particulièrement aux aspects «détails intéressants » et "informations objectives » en présentant le tableau 5 qui indique le pourcentage d'élèves ayant incorporé à leur texte les divers procédés explicatifs, avant et après la mise en œuvre de la séquence didactique.

Tableau 5. Pourcentages d'élèves appartenant aux deux groupes ayant eu recours à des procédés de développement d'une idée au pré-test et au post-test.

\begin{tabular}{|l|l|l|l|l|}
\hline & \multicolumn{2}{l|}{$\begin{array}{l}\text { Groupe } \\
\text { expérimental }\end{array}$} & \multicolumn{2}{l|}{ Groupe témoin } \\
\hline Procédés pour développer une idée & Prétest & Post-test & Prétest & Post-test \\
\hline Description & $86 \%$ & $92 \%$ & $100 \%$ & $98 \%$ \\
\hline Données chiffrées & $61 \%$ & $89 \%$ & $69 \%$ & $67 \%$ \\
\hline Citation & $0 \%$ & $5 \%$ & $0 \%$ & $0 \%$ \\
\hline Exemples & $0 \%$ & $28 \%$ & $0 \%$ & $0 \%$ \\
\hline Projection & $2 \%$ & $2 \%$ & $0 \%$ & $0 \%$ \\
\hline Supposition & $2 \%$ & $17 \%$ & $3 \%$ & $2 \%$ \\
\hline
\end{tabular}

Le tableau 5 permet de constater, d'une part, que les élèves du groupe expérimental ont employé, au post-test, une plus grande variété de procédés de développement des idées que les élèves du groupe témoin et, d'autre part, que les progrès les plus importants se situent au niveau des données chiffrées et des exemples puisque le pourcentage d'élèves qui 
a utilisé ces procédés dans leur texte a augmenté de $30 \%$ entre le pré-test et le post-test. On remarque également que le progrès le moins important concerne le recours aux citations avec une augmentation de $5 \%$.

\section{Discussion des résultats}

Dans l'ensemble, les résultats positifs obtenus dans toutes les dimensions de la cohérence textuelle viennent confirmer l'efficacité d'une intervention axée sur l'enseignement de plusieurs stratégies conçues pour amener l'élève à mobiliser les opérations de haut niveau qui sous-tendent l'écriture d'un texte de comparaison. Toutefois, même si les progrès réalisés par les élèves $d u$ groupe expérimental sont généralisés, il existe néanmoins quelques variations dans les résultats qui méritent une interprétation afin de mieux comprendre la nature spécifique de ces progrès et de dégager quelques pistes pour améliorer la séquence sur le texte explicatif de comparaison.

Si l'on compare les progrès réalisés dans les trois dimensions de la cohérence textuelle, le tableau 4 montre que c'est dans la dimension macrostructurelle que les élèves ont fait le plus grand progrès. L'examen des différences moyennes pour chacun des aspects à l'intérieur de cette dimension indique que les deux progrès les plus importants se situent aux niveaux du paragraphe des ressemblances et des transitions entre les paragraphes. Pour ce qui est du paragraphe des ressemblances, les données brutes révèlent que la hausse dans la moyenne est due au fait, qu'au pré-test, seulement 2 élèves sur 40 ont traité des ressemblances dans leur texte, alors qu'au post-test, 38 élèves l'ont fait. Concernant les transitions, la hausse s'explique par le fait, qu'au pré-test, la moitié des élèves ont omis les phrases de transition, tandis qu'au post-test, cette même moitié d'élèves s'est mise à employer systématiquement des phrases telles que : Premièrement, je commencerai [ou je vais vous présenter] par les différences [ou par les ressemblances]; Les deux villes comprennent également des différences par rapport au [...] ou Tout d'abord, la première différence concerne [...] pour marquer explicitement le passage d'un paragraphe à l'autre selon la structure comparative.

Viennent ensuite, en ordre d'importance, les progrès au niveau de la conclusion, de l'introduction et du paragraphe des différences. Pour la conclusion, la hausse dans les résultats au post-test s'explique comme suit: au pré-test, la conclusion, présente dans 16 copies seulement, a été jugée partiellement réussie, soit parce qu'il manquait une composante (en général, l'ouverture), soit parce que le lien avec le sujet du texte n'était pas évident, tandis qu'au post-test, la conclusion était présente dans 34 copies dont 11 qui ont reçu le score maximal, car les élèves ont réussi à intégrer les deux composantes requises (le résumé et l'ouverture), et 23 qui ont reçu la mention « réussite partielle » pour les mêmes raisons que celles invoquées dans le cas du pré-test.

En ce qui concerne l'introduction, les données brutes indiquent que l'écart entre le prétest et le post-test provient du fait qu'au pré-test, seulement 26 élèves ont fait une introduction alors qu'au post-test, la totalité des élèves en a fait une. Toutefois, parmi ces derniers, 16 d'entre eux seulement ont répondu à toutes les exigences en intégrant les trois composantes requises, soit le sujet amené, posé et divisé. Quant aux 24 autres élèves, leur performance a été qualifiée, comme dans le cas de la conclusion, de réussite partielle, car ils n'ont pas incorporé de phrase servant à amener le sujet. À ce propos, il est intéressant de noter qu'en général, les élèves qui ont omis d'amener le sujet dans 
l'introduction, ont également omis de terminer leur conclusion par une phrase d'ouverture du sujet. Or, ces deux activités font toutes les deux appel à la même opération de hiérarchisation conceptuelle qui exige de prendre du recul pour faire un lien entre le sujet et un contexte plus général. Selon l'enseignante, pour éviter la surcharge cognitive, il serait préférable de travailler sur ces deux composantes textuelles plus tard dans la scolarité des élèves.

Finalement, pour ce qui est du paragraphe des différences, la hausse au post-test résulte du fait qu'au pré-test, 13 élèves (par opposition à 4 au pos-test) n'ont tout simplement pas traité des différences dans leur texte et 17 élèves seulement (par opposition à 31 au posttest) avaient au moins deux aspects qui ont fait l'objet d'une comparaison et qui comprenaient des éléments touchant les deux termes de la comparaison. À ce propos, aux dires de l'enseignante, les élèves plus faibles ont eu besoin de beaucoup plus de soutien que les autres pour saisir la notion d'aspect et distinguer les aspects des éléments servant à les développer.

En somme, l'interprétation des variations dans les différences moyennes entre le pré-test et le post-test pour ce qui est de la dimension macrostructurelle suggère que les élèves du groupe expérimental sont passés d'une représentation mentale partielle de la structure du texte de comparaison à une représentation beaucoup plus complète qui les aurait guidés à toutes les étapes du processus d'écriture. Comme dans le cas de recherches antérieures portant sur d'autres types de texte (Broer, Kieviet \& Leewe, 2002 ; Hammann \& Stevens, 2003; Troia, 2006; Cavanagh, 2006, 2009), ce changement peut, certes, être attribué à l'effet de l'exploitation du schéma du texte explicatif de comparaison dans diverses activités d'analyse de textes ainsi que lors de la planification et de la révision de leur propre texte.

Le deuxième progrès le plus important se situe au plan de la cohérence situationnelle. L'examen des différences moyennes pour chacun des aspects à l'intérieur de cette dimension, permet de noter que les deux progrès les plus importants se manifestent par une capacité accrue chez les élèves à tenir compte des contraintes inhérentes à la situation d'écriture et à baser leur explication sur des informations objectives, ce qui met en évidence l'intérêt d'enseigner l'emploi d'une stratégie d'analyse de la situation d'écriture ("Examiner à la loupe ») en combinaison avec des stratégies pour se donner un but quant aux informations recherchées («Cibler l'information»), pour retenir les documents pertinents en fonction du but établi («Survoler l'information») et pour prendre des notes («Noter l'essentiel »). L'observation des traces de surlignage dans les pochettes informatives au pré-test et au post-test confirme que ces stratégies ont conduit les élèves du groupe expérimental à sélectionner le contenu des textes sources en fonction des exigences liées à la tâche de comparer deux réalités entre elles. Les élèves du groupe témoin, qui n'ont pas bénéficié de l'enseignement de ces stratégies, ont procédé quant à eux, au pré-test comme au post-test, à une sélection très aléatoire des informations contenues dans les textes présentés dans les pochettes.

Quant au changement dans la capacité des élèves à développer une explication en s'appuyant sur des faits objectifs, il peut sans doute être attribué à l'incorporation de plusieurs procédés explicatifs dans les textes modèles que les élèves ont analysés et à l'enseignement de la stratégie «DIP » au moment de la mise en texte. Le tableau 5 montre toutefois que l'intégration des citations comme moyen de donner une crédibilité aux explications fournies gagnerait à être davantage explicitée lors d'une intervention ultérieure. Enfin, le progrès le moins important concerne l'emploi d'un vocabulaire varié 
et précis pour donner au texte de comparaison un ton objectif, ce qui n'est pas étonnant étant donné le milieu linguistique minoritaire dans lequel évoluent les élèves qui ont participé à cette étude. Ce résultat, qui corrobore les résultats d'une recherche menée sur l'écriture du récit (Cavanagh, 2009), souligne la nécessité de consacrer plus de temps à l'enrichissement des connaissances lexicales.

51 Finalement, viennent les progrès au plan de la cohérence microstructurelle. En comparant les différences moyennes entre elles pour le groupe expérimental, on remarque que le changement le plus important se situe au niveau de la capacité des élèves à assurer la continuité des idées et que le changement de moins grande envergure touche leur habileté à expliciter le rapport entre les idées. Concernant la continuité, l'observation des copies du post-test révèle une tendance chez les élèves à privilégier la répétition du même terme comme moyen de reprendre le sujet de la phrase précédente (par exemple : Au soccer, le ballon est rond; au football, le ballon a une forme ovale). Vient ensuite, mais dans une proportion moindre, le recours aux pronoms (par exemple: Enfin, la reine était représentée deux fois. À Montréal, elle est venue en personne, mais à Vancouver la Gouverneure générale du Canada la représentait) et, en dernier lieu - mais très rarement, et seulement chez les plus forts - l'emploi de synonymes (par exemple: Au contraire, à Edmonton il fait environ -30 degrés [en hiver], alors quand on est dans cette température, il faut bien s'habiller) ou d'une périphrase (par exemple : Les Jeux Olympiques de Montréal étaient en 1976... En 2010, les Jeux étaient à Vancouver...Ces deux jeux avaient des points en communs... Les coûts étaient très hauts pour ces deux évènements sportifs mondiaux). Ce résultat suggère que la stratégie " Apporter avec moi », n'a pas conduit les élèves à recourir à une variété de procédés linguistiques pour assurer la continuité des idées dans le cadre d'une organisation textuelle qui présente, en alternance, les différents aspects de deux objets. En effet, l'enseignante a indiqué lors des entretiens qu'elle avait consacré peu de temps à l'emploi de cette stratégie. En plus de passer plus de temps sur cette stratégie, il faudrait probablement incorporer à la séquence didactique, au moment de la révision, des activités décontextualisées visant à approfondir cette notion spécifique à partir d'exemples tirés des textes des élèves.

52 En ce qui a trait à la capacité des élèves à exprimer la relation de comparaison entre deux éléments, l'observation des copies permet de dégager deux tendances. Chez les élèves du groupe témoin (pré-test et post-test), le recours aux connecteurs ou aux expressions de comparaison est très limité et, typiquement, les élèves n'évoquent pas les deux termes de la comparaison dans la même phrase. Ils se contentent d'affirmer qu'il y a une différence (dans la dimension des terrains de jeux, l'équipement de protection, etc.) ou une ressemblance (esprit sportif, nombre de francophones, etc.) entre les deux termes de la comparaison. Ensuite, ils décrivent chaque terme séparément, comme dans les deux exemples suivants: 1) L'équipement de protection est différent. Au soccer, on porte des jambières. Au football on porte un casque et 2) Les différences entre Edmonton et Vancouver sont les suivantes: Edmonton est entouré de prairies. Vancouver est à côté de l'océan. Tout au plus, la relation de comparaison entre deux éléments est marquée par un mais, comme dans l'exemple suivant: En soccer tu peux faire un but juste d'une façon mais en football américain il y a trois façons.

53 Par contre, chez les élèves du groupe expérimental, on remarque, dans les productions du post-test, un recours à une plus grande variété de connecteurs ou d'expressions pour marquer la comparaison et une tendance à évoquer les deux termes de la comparaison dans une même phrase. Ainsi, dans le paragraphe traitant des différences on trouve des 
comparatifs pour signaler une relation d'infériorité ou de supériorité (par exemple: Edmonton a le West Edmonton Mall et Vancouver a quelque chose de similaire mais moins grand ), ou une relation d'égalité (par exemple: La mer est une des principales attractions de Vancouver, mais Edmonton utilise la technologie pour créer une expérience toute aussi amusante) ou encore une relation d'opposition à l'aide de connecteurs tels que, par contre, alors que, contrairement à, etc. (par exemple: Ensuite, il y a eu les controverses. À Montréal, c'était les dépenses trop élevées et le manque d'organisation pour construire le stade olympique. Tandis qu'à Vancouver, cétait le manque de français à plusieurs moments durant ces jeux.). Dans le paragraphe des ressemblances, le recours à l'expression les deux est fréquent (par exemple: Dans les deux villes, 30000 des habitants sont francophones et parlent la langue française.).

Le constat de l'augmentation de la capacité des élèves à expliciter des rapports de comparaison, souligne l'intérêt de leur fournir une banque de procédés linguistiques spécifiques qui supportent la structure de comparaison, de leur faire observer ces procédés dans le contexte des textes analysés au début de la séquence et de leur montrer comment les utiliser, particulièrement au moment du modelage des stratégies de mise en texte et de révision. Dans le but de soutenir davantage les élèves plus faibles, il serait sans doute avantageux d'incorporer au dispositif une stratégie qui associerait les connecteurs de comparaison au schéma, comme nous l'avons d'ailleurs fait dans le cas de l'enseignement du texte d'opinion (Cavanagh, 2005 : 123).

Au-delà du constat de ces progrès au niveau de la cohérence textuelle, les quatre entretiens conduits avec l'enseignante ont apporté un éclairage sur certaines différences entre les élèves forts et les élèves faibles en ce qui concerne leur emploi des stratégies enseignées. Ainsi, contrairement aux élèves forts, les élèves faibles ont eu de la difficulté à comprendre ce qui distinguait les stratégies entre elles et, par conséquent, ils ont eu tendance à percevoir certaines stratégies comme étant inutiles. En particulier, comme la différence entre la stratégie "Survoler l'information » et "Noter l'essentiel » n'était pas claire pour eux, ils ont eu tendance à conclure que le travail exigé par la seconde stratégie était redondant. À cette difficulté, s'est ajoutée celle à percevoir les liens qui unissent les stratégies entre elles, ce qui a conduit les élèves plus faibles à traiter chaque stratégie comme une entité séparée et à procéder de façon linéaire sans retour en arrière pour vérifier certaines informations, se les rappeler ou établir des liens entre elles. Au contraire, les élèves forts, qui semblaient être plus conscients des liens entre les stratégies, ont fait preuve, à la manière du scripteur habile (Hayes, 1995), d'une plus grande récursivité et d'une meilleure autorégulation. Enfin, selon le témoignage de l'enseignante, la difficulté pour certains élèves à percevoir les ressemblances et les différences entre les stratégies pourrait résulter, du moins en partie, d'une faiblesse au niveau du vocabulaire associé à l'emploi de la stratégie.

\section{Conclusion}

L'objectif de cette étude était d'évaluer l'efficacité d'une séquence didactique adaptée à l'écriture d'un texte explicatif de comparaison sur la capacité d'élèves franco-albertains de 12 ans à composer des textes explicatifs cohérents. Pour ce faire, un devis pré-test, post-test avec groupe témoin a été utilisé. L'analyse des résultats quantitatifs indique que les élèves ont progressé dans les trois dimensions de la cohérence textuelle (voir des exemples de productions écrites à l'annexe 1). En dépit de certaines limites 
méthodologiques (par exemple, la taille restreinte de l'échantillon) ce résultat confirme l'intérêt d'outiller les élèves en stratégies spécifiques au type de texte visé.

En même temps cependant, l'interprétation de certaines variations dans les résultats des élèves souligne l'importance d'incorporer au dispositif des activités décontextualisées pour que les élèves apprennent à recourir à une plus grande variété de procédés de progression et de continuité au moment de la mise en texte. Néanmoins, pour éviter la surcharge cognitive, il serait important de répartir ces notions à travers quelques années successives. Par ailleurs, l'analyse des données qualitatives issues des entretiens avec l'enseignante a révélé l'importance, surtout pour les élèves plus faibles, de faire valoir l'utilité de chaque stratégie tout en explicitant le lien qui unit les stratégies entre elles, de s'assurer que les élèves comprennent le métalangage auquel elles sont associées et de montrer leur gestion active et récursive par le recours à des stratégies d'autorégulation.

Cette recherche ouvre la voie à plusieurs recherches futures. Premièrement, il conviendrait d'expérimenter le même genre de séquence pour le texte explicatif, mais en mettant l'accent sur la structure problème-solution et sur la structure cause-effet. Une telle étude permettrait de conceptualiser les défis particuliers que présente chacune de ces structures pour les élèves. Deuxièmement, étant donné que les classes en milieu minoritaires sont très hétérogènes, avec K.-R. Harris, S. Graham, M. Brindle et K. Sandmel (2009), nous croyons qu'il serait profitable de mener une recherche longitudinale pour voir comment des élèves de niveaux d'habiletés variés en écriture s'approprient les stratégies enseignées. Troisièmement, vu que les élèves francophones en milieu linguistique minoritaire sont généralement peu motivés à écrire, en partie en raison d'un manque de confiance dans leurs habiletés, il serait pertinent d'étudier l'impact des séquences portant sur le texte explicatif sur la motivation à écrire de ces élèves. Finalement, à la suite de G.-A. Troia (2006), il apparait crucial de vérifier dans quelle mesure les apprentissages réalisés dans le cadre du texte de comparaison se maintiennent à long terme et, dans cette perspective, de se pencher sur le développement de la capacité des élèves à transférer les stratégies de lecture et d'écriture enseignées dans le contexte d'autres disciplines, voire même d'autres langues.

\section{BIBLIOGRAPHIE}

ADAM J.-M. (1999) : Les schémas. Un langage transdisciplinaire, Paris , Éd. L'Harmattan.

- (2005) : Les textes : types et prototypes. Récit, description, argumentation, explication et dialogue, $2^{\mathrm{e}}$

éd., Paris, A. Colin.

- (2008) : La linguistique textuelle. Introduction à l'analyse des discours, $2^{\mathrm{e}}$ éd., Paris, A. Colin.

ALBERTA LEARNING (1998) : Programme d'études de français - langue maternelle. Edmonton, Alberta

Resources Branch.

APotheloz, D. \& mieville D., avec la collab. de J.-I. Grize (1989) : « Cohérence et discours

argumenté », in : M. Charolles (dir.), The Resolution of Discourse Processing. Coherence or Consistency

Dissonances, Hamburg, H. Buske Verlag, p. 68-87. 
BALL, A. (2006) : « Teaching writing in Culturally Diverse Classrooms », in : C. A. MacArthur, S. Graham \& J. Fitzgerald (dirs), Handbook of Writing Research, New York, Guildford Press, p. 293-310. BEARD EL-DINARY, P. \& SCHUDER, T. (1993) : « Seven Teachers Acceptance of Transactional Strategies Instruction during Their First Year Using It », The Elementary School Journal, 94 (2), p. 207-219.

BLAIN, S. (2001) : « Study of Verbal Peer Feedback on The Improvement of the Quality of Writing and the Transfer of Knowledge in Francophone Student in Grade 4 Living in a Minority Situation in Canada ", Language, Culture and Curriculum, 14 (2), p. 156-170.

BLAIN, S. \& LAFONTAINE, L. (2010) : « Mettre les pairs à contribution lors du processus d'écriture : une analyse des impacts du groupe de révision rédactionnelle sur le transfert des connaissances en orthographe grammaticale dans différents contextes linguistiques ", Revue des sciences de l'éducation, 36 (2), p. 469-491.

BLAIN, S. \& PAINCHAUD, G. (1999) : « L'impact de la rétroaction verbale des pairs sur l'amélioration des compositions des élèves de $5^{\mathrm{e}}$ année en immersion française ", The Canadian Modern Language Review/La revue canadienne des langues vivantes, 56 (1), p. 73-98.

BOUdREAUlt, P. (2000) : « La recherche quantitative », in : T. Karsenti \& L. Savoie-Zajc (dirs), Introduction à la recherche en éducation, Sherbrooke, CRP, p. 141-170.

BRASSART, D. (1998) : « Approches cognitives de la didactique de la composition écrite », Psychologie \& Éducation, 33, p. 13-29.

Broer, N. A., Aarnoutse, C. A. J., Kieviet, F. K. \& Leeuwe, J. F. J. van (2002) : « The Effect of Instructing the Structural Aspect of Texts », Educational Studies, 28 (3), p. 213-238.

CARTER-THOMAS, S. (2000) : La cohérence textuelle. Pour une nouvelle pédagogie de l'écrit, Paris, Éd. L' Harmattan.

CARTIER, S. (2007) : Apprendre en lisant au primaire et au secondaire: Mieux comprendre et mieux intervenir, Anjou (Québec), CEC.

CAVANAGH, M. (2005) : Stratégies pour écrire un texte d'opinion, Montréal, Chenelière Éducation (coll. « Didactique »).

- (2006) : «Validation d'un programme d'intervention pour la cohérence des écrits argumentatifs au primaire », Revue des sciences de l'éducation, 32 (1), p. 159-182.

- (2007a) : « Profil scriptural d'élèves franco-albertains du primaire en vue de l'implantation d'un programme d'intervention en écriture ", Revue canadienne de l'éducation/Canadian Journal of Education, 30 (3), p. 691-724.

- (2007b) : Stratégies pour écrire un récit imaginaire, Montréal, Chenelière Éducation (coll. « Didactique »).

- (2009) : « Vers un programme d'intervention pour aider les élèves francophones de 9-10 ans en milieu minoritaire à rédiger un récit cohérent, Éducation francophone en milieu minoritaire, 3 (1), p. 1-14.

- (2010a) : Stratégies pour écrire un texte explicatif, Montréal, Chenelière Éducation (coll. « Didactique »).

- (2010b) : « Élaborer une séquence didactique à l'écrit : selon quels principes théoriques ? », Enjeux, 77, p. 83-110.

CAVANAGH, M. \& BLAIN, S. (2009) : « Relever quatre défis de l'enseignement du l'écrit en milieu francophone minoritaire », Les Cahiers franco-canadiens de l'Ouest, 21 (1-2), p. 151-178.

CHAROLLES, M. (1978) : «Introduction aux problèmes de la cohérence des textes. Approche théorique et études des pratiques pédagogiques ", Langue française, 38, p. 7-41. 
- (2002) : La référence et les expressions référentielles en français. Paris, Éd. Ophrys.

- (2006) : « De la cohérence à la cohésion du discours », in : Cohérence et discours, éd. par F. Calas, Paris, Presses de l'université Paris-Sorbonne, p. 25-38.

CONSEIL DES MINISTRES DE L'ÉDUCATION DU CANADA/CMEC (2004) : Résultats pancanadiens des élèves francophones en milieu minoritaire au Programme d'indicateurs du rendement scolaire (PIRS), Rapport analytique, Toronto, CMEC.

CORMIER, M. (2005) : La pédagogie en milieu minoritaire francophone : une recension des écrits, Ontario, FCEE.

DICKSON, S. (1999) : « Integrating Reading and Writing to Teach Compare-Contrast Text Structure : A Research-Based Methodology ", Reading et Writing Quarterly, 14, p. 49-79.

DE WECK, G. (1991) : La cohésion dans les textes d'enfants : étude du développement des processus anaphoriques, Paris, Delachaux et Niestlé.

EVA [groupe] (1996), De l'évaluation à la réécriture. Réécrire au cycle III, Paris, Hachette/INRP. - (1991) : Évaluer les écrits à l'école primaire, Paris, Hachette/INRP.

FALARDEAU, M. \& LORANGER M. (1993) : « Le choix de stratégies d'apprentissage dans différents contextes scolaires par l'élève du primaire et du secondaire ", Revue canadienne de l'éducation, 18, p. 307-322.

GAGNON, R. \& ZIARKO H. (2009) : « Apprendre à écrire un texte documentaire de comparaison en $2^{e}$ année du primaire : étude comparée d'interventions didactiques contrastées ", Revue des sciences de l'éducation, 35 (3), p. 127-148.

GARCIA-DEBANC, C. (1988) : «Propositions pour une didactique du texte explicatif », Aster, 6, p. 129-163.

GARCIA-DEBANC, C., PLANES, L. \& ROGER, C. (2004) : Objectif écrire, $3^{\mathrm{e}}$ éd., Scéren, CRDP LanguedocRoussillon.

GIASSON, J. (2007) : La compréhension en lecture, 3e éd., Montréal, De Boeck [1990].

GRAHAM, S. (2006) : « Strategy Instruction and the Teaching of Writing : A Meta-analysis », in : C. A. MacArthur, S. Graham \& J. Fitzgerald (dirs), Handbook of writing research, New-York, Guilford Press, pp. 187-207.

GRAHAM, S. \& HARRIS, K.-R (2003) : Students with Learning Disabilities and the Process of Writing : A Meta-Analysis of SRSD Studies, in : H. L. Swanson, K. R. Harris \& S. Graham (dirs), Handbook of learning disabilities, New-York, Guilford Press, p. 323-344.

GRAHAM, S. \& Perin, D. (2007) : Writing next: Effective strategies to improve writing of adolescents in middle and high schools, Washington, Alliance for Excellent Education.

GRENIER, S. (2008) : Dazibao : référentiel grammatical, Québec, Groupe Modulo.

HAMMANN, L.-A. \& STEVENS R.-J. (2003) : Instructional Approaches to Improving Students' Writing of Compare-Contrast Essay, Journal of Literacy Research, 35, p. 731-756.

HAYES, J. (1995) : « Un nouveau modèle du processus d'écriture », in : J.-Y. Boyer, J.-P. Dionne \& P. Raymond (dirs), La production de textes. Vers un modèle d'enseignement de l'écriture, Montréal, Éd. Logiques, p. 49-72.

- (1998) : « Un nouveau cadre pour intégrer cognition et affect dans la rédaction », in : A. Piolat \& A. Pélissier (dirs), Rédaction de textes. Approche cognitive, Lausanne, Delachaux et Niestlé, p. 51-101. 
- (2004) : “What triggers revision ?", in : L. Allal. Chanqouy \& P. Largy (dirs), Volume 13, Revision : Cognitive and Instructional Processes, Boston, Kluwer, p. 9-20.

HARRIS, K.-R., GRAHAM., S. BRINDLE M. \& SANDMEL K. (2009) : Metacognition and Children's Writing, in : D. J. Hacker, J. Dunlosky \& A.C. Graesser (dirs), Handbook of Metacognition in Education, Mahwah, L. Erlbaum.

HENSLER, H. (1992) : Élaboration d'une conception de l'enseignement et de la formation des enseignants dans le domaine des stratégies d'apprentissage, thèse de doctorat, université de Montréal.

KARMiloff-SMith, A. (1992) : Beyond Modularity. A Developmental Perspective on Cognitive Science, Cambridge, MIT Press.

LAPARRA, M. (1986) : « Analyse des difficultés des élèves en matière de lecture et d'écriture des textes explicatifs ", Pratiques, 51, p. 77-85.

LUNDQUIST, L. (1980) : La cohérence textuelle : syntaxique, sémantique, pragmatique, Kobenham, Nyt Nordisk Forlag Arnold Busck.

MACARTHUR, C.-A. \& PHILIPPAKos, Z. (2010) : «Instruction in a Strategy for Compare-Contrast Writing », Exceptional Children, 76 (4), p. 438-457.

MEYER, B.-J.-F. (1985) : « Prose Analysis : Purpose, Procedure and Problems », in : B. K. Britton \& J. B. Black. (dirs), Understanding expository text, Hillsdale, L. Erlbaum.

OGLE, D. (1986) : « K-W-L : A Teaching Model That Develops Active Reading of Expository Text », The Reading Teacher, 39 (6), p. 564-570.

PIGALLET, P. (1996) : Méthodes et stratégies de lecture: Pour un art de lire, Paris, ESF.

RICHGELS, D.-J., MCGEE, L.-M., LOMAX R.-G. \& SHEARD C. (1987) : « Awareness of Four Text Structures : Effects on Recall of Expository Text, Reading Research Quaterly, 22, p. 177-196.

ROBERT, A. (2001) : « Écrire des textes explicatifs : passage à l'acte », « Grand N », 68, p. 91-113.

SPIVEY, N.-N. (1991) : « The Shaping of Meaning : Options in Writing Comparison », Research in the Teaching of English, 25, p. 390-418.

TARDIF, J. (1992) : Pour un enseignement stratégique. L'apport de la psychologie cognitive. Montréal, Éd. Logiques.

- (1999) : Le transfert des apprentissages, Montréal, Éd. Logiques.

TROIA, G.-A. (2006) : « Writing Instruction for Students with Learning Disabilities », in: C.A.

MacArthur, S. Graham \& J. Fitzgerald (dirs), Handbook of Writing Research, New York, Guildford Press, p. 324-336.

VYGoTsky, L. (1978) : Mind in Society: The development of Higher Psychological Processes, Cambridge, Harvard University Press.

WONG, B. (2000) : «Writing Strategies Instruction for Expository Essays for Adolescents with and without Disabilities », Topics in Language Disorders, 20, p. 29-44.

\section{ANNEXES}




\title{
Annexe 1. Exemples de productions écrites représentatives des progrès réalisés dans les trois dimensions de la cohérence textuelle
}

\author{
Situation d'écriture sur le football (Prétest)
}

Cette année, le monde entier a eu le regard tourné vers l'Afrique du Sud pour encourager son équipe de soccer lors de la FIFA. Pendant un repas de famille, le sujet de la coupe du monde est abordé. Ta tante soutient alors que toutes les sortes de « football » sont pareilles : deux équipes qui essayent de mettre un ballon dans un but ! L'ignorance de ta tante te dérange et tu te donnes comme mission de lui expliquer par écrit les ressemblances et les différences entre le football américain et le soccer afin qu'elle en connaisse un peu plus sur le sujet.

\section{Texte écrit par Marc (élève fort) lors du pré-test (groupe expérimental)}

Lors du dernier repas de famille, ma chère tante, vous aviez dis qu'il y a peu de différences entre le Football américain et le soccer. Je crois que vous avez tord, car premièrement, le soccer est en sorte l'ancêtre du Football (le soccer date environ du $15^{\mathrm{e}}$ siècle et le Football d'environ vers 1870). Les règles du Football viennent du Rugby, alors ce sont des règles qui ressemblent peu au Soccer. Par exemple on ne peut pas toucher au ballon avec les mains dans le soccer, tandis qu'il est primordial de le faire au Football. Encore au Football, il y a trois façons de faire un but (Le "Touchdown", Un "Fieldgoal" ou le "Safety") et vous savez sûrement qu'il n'y a qu'une façon de le faire au soccer.

Et puis, il y a la grandeur du terrain, la forme de la balle et surtout l'équipement. On croirait que les joueurs de football s'en vont en guerre avec tout leur équipement! Ils ont des casques avec une grille à l'avant et des épaulières, comparé au joueurs de soccer, ils ressemblent à des lutteurs!

Vous avez plus de chance de tomber sur quelqu'un qui connais le soccer, car c'est un sport mondialement connu. Mais si vous êtes en Amérique du nord, vous verrez que le Football est un sport apprécié dans ce coin du monde.

Enfin, je ne dis pas que vous avez tord de penser que c'est sports se ressemblent. C'est un préjugé connue. Quand j'étais plus jeune j'aurais dis la même chose. Car si il y a une ressemblance entre ces deux sports, c'est qu'en Europe ils s'appellent tous les deux "Football".

\section{Situation d'écriture sur deux villes canadiennes (post-test)}

Cet été, tu es allé en vacances au Québec. Là-bas, tu as rencontré Francine, une fille de ton âge dont les parents veulent déménager dans l'Ouest canadien. La semaine dernière, Francine t'a écrit une lettre dans laquelle elle te dit que ses parents hésitent entre deux villes : Edmonton (en Alberta) ou Vancouver (en Colombie-Britannique). Tu décides alors d'écrire à ton amie pour lui expliquer les ressemblances et les différences entre ces deux villes. 


\section{Texte écrit par Jessica (élève faible) lors du post-test (groupe expérimental)}

Vancouver ou Edmonton?

Chère Francine,

Aujourd'hui je vais te parler des ressemblances et des différences entre Edmonton et Vancouver.

Commençons par les ressenblances. Pour débuter, Edmonton et Vancouver on à peu près le même nombre de Francophones qui compte a peu près 30000 personnes «qui équiveaux au quard d'Edmonton». De plus ses deux villes on presque le même nombre d'autochtone qui vivent et qui son accepté dans la communauter. Enfin l'Alberta et la Colombie-Britannique on tout les deux des villes principales don Edmonton et Vancouver.

Allons dans les différences maintenant. En premier, Edmonton est une ville asser froides. En janvier, il fait $-28^{\circ} \mathrm{C}$ à $-33^{\circ} \mathrm{C}$ la plus pare du temp. Vancouver de l'autre coter est beaucoup plus chaud avec des $+10^{\circ} \mathrm{C}$ à $12^{\circ} \mathrm{C}$. Par exemple, Edmonton est beaucoup plus ensoleiller et peut avoir qu'un nuage dans le ciel. Mais Vancouver a plutôt de la pluie et beaucoup de nuages qui fait que sa pourait être fatiguant de regarder le ciel à ce temp ci de l'année. Deuxièmement, Edmonton ajoute la langue chinoise l'orsque tu voudras appeler le 911. Inci tu pourra dire ce qui ya de mal avek toi en chinois. Par contre, Vancouver avait cette possibiliter bien avant que Edmonton le face. Dernièrment, Edmonton est dans le centre des plaines et est éloigné des montagnes. Vancouver est plutôt au bor des montagnes et est éloigné des plaines alors ceci est ton choix si tu veux vivre dans les plaines ou dans les montagnes avec tes parents.

Je t'ai parlé des différences et des ressemblances entre Edmonton et Vancouver. Moi, je trouve que tes parents devrait choisir Vancouver mais ceci est leur choix et pas le mien. Une Question, penses-tu que Québec serait meilleur que la Colombie-Britannique?

Sincèrement,

Ton amie Jessica

\section{NOTES}

1. Pour une description détaillée des scénarios de modelage, voir Cavanagh, 2010a.

2. ${ }^{*} \mathrm{p}<0,05 * * \mathrm{p}<0,01 * * * \mathrm{p}<0,001$.

3. ${ }^{*} \mathrm{p}<0,05 * * \mathrm{p}<0,01 * * * \mathrm{p}<0,001$.

\section{RÉSUMÉS}

Cette étude vise à évaluer l'impact d'une séquence didactique privilégiant l'enseignement de stratégies sur la capacité d'adolescents franco-canadiens vivant en milieu linguistique minoritaire à rédiger un texte explicatif de comparaison. Plus spécifiquement, à l'aide d'un 
design pré-test et post-test appliqué à un groupe expérimental $(\mathrm{n}=40)$ et à un groupe contrôle $(n=41)$, l'étude a vérifié dans quelle mesure la séquence a permis aux élèves de composer des textes explicatifs de comparaison cohérents. Trois dimensions de la cohérence textuelle ont été évaluées, à savoir macrostructurelle, microstructurelle et situationnelle. Les résultats montrent que les élèves ont progressé de façon significative dans chacune de ces dimensions, ce qui confirme l'intérêt d'outiller les élèves en stratégies spécifiques au type de texte visé.

The aim of this study is to evaluate the impact of a didactic sequence centred on the teaching of strategies on the ability of a group of Franco-Canadian adolescents living in a linguistic minority situation to write a comparative expository text. More specifically, using a pretest post-test design with an experimental group $(n=40)$ and a control group $(n=41)$, the study verifies to what extent the sequence allowed students to compose coherent expository texts. Three dimensions of textual coherence were evaluated, namely macrostructural, microstructural and situational coherence. The results show that the students progressed significantly in each of these dimensions and this finding confirms the usefulness of equipping students with strategies specific to the type of text being taught.

\section{INDEX}

Mots-clés : pédagogie en milieu minoritaire, type de texte, texte explicatif de comparaison, stratégies d'écriture, cohérence textuelle

Keywords : pédagogy in minority communities, type of text, explanatory text for comparing, write strategies, textual coherence

\section{AUTEUR}

\section{MARTINE CAVANAGH}

University of Alberta 